\title{
The Effect and the Mechanism of Comprehensive Treatment on the Ocular Surface and the Visual Quality of Online Teachers with a Mild-to-Moderate Dry Eye Condition During the Early Phase of Coronavirus Disease 2019
}

\section{Yan-Xi Wang \\ Gang-Jin Kang \\ Juan Zhou \\ Kai-Ming Li \\ Man-Hua Xu}

Department of Ophthalmology, Affiliated Hospital of Southwest Medical University, Luzhou, Sichuan, People's Republic of China
Correspondence: Gang-jin Kang Department of Ophthalmology, Affiliated Hospital of Southwest Medical University, Luzhou, Sichuan, People's Republic of China

Tel +86I380828I600

Email drkangjgtt@I63.com
Objective: To investigate the effect of comprehensive treatment on ocular surface function and the visual quality of online teachers with a mild-to-moderate dry eye condition during the early phase of coronavirus disease 2019 (COVID-19).

Methods: Secondary school online teachers diagnosed with a mild-to-moderate dry eye disease in our outpatient clinic from February to May 2020 were enrolled in this retrospective cross-section study, and all patients received dry eye comprehensive treatment. A questionnaire survey on eye-use habits, visual quality and dry eye-related indicators was collected before and after treatment ( 2 and 4 weeks). The changes and the correlations between indicators before and after treatment were compared.

Results: A total of 30 patients ( 15 females and 15 males) were included. After comprehensive treatment, patients had significantly higher central tear meniscus height (TMH), non-invasive first tear film breakup time (NIBUTf) and non-invasive average tear film breakup time (NIBUTav) than those before with statistical significance $(\mathrm{P}<0.05)$. Lower ocular surface disease index (OSDI) and Meibomian gland scores were observed after treatment with statistical significance $(\mathrm{P}<0.05)$. Objective scatter index $(\mathrm{OSI})$, modulation transfer function $(\mathrm{MTF})$ cutoff, strehl ratio (SR), and tear film objective scatter index (TF-OSI) were significantly improved after treatment $(\mathrm{P}<0.05)$. Besides, TF-OSI was positively correlated with the changes in OSDI, Meibomian gland score, eye-use duration and OSI with statistical significance $(\mathrm{P}<$ 0.05), while it was negatively correlated with NIBUTf, NIBUTav, the TMH of the central lower eyelid, SR, sleep duration, conjunctival congestion and the MTF cutoff $(\mathrm{P}<0.05)$, respectively. No correlation between TF-OSI and ciliary congestion was found $(\mathrm{P}>0.05)$.

Conclusion: Comprehensive treatment could effectively improve the symptoms and visual quality of online teachers with a mild-to-moderate dry eye condition during the early stage of COVID-19 pandemic.

Keywords: coronavirus disease 2019 pandemic, online teacher, dry eye, comprehensive treatment, visual quality

\section{Introduction}

Dry eye is a common multifactorial inflammatory disease that can lead to chronic eye discomfort, visual impairment, and reduced quality of life, which is increasingly induced by environmental pollution and the common use of electronic 
devices. ${ }^{1}$ Besides, dry eye is a common chronic eye disease after refractive error and a chronic condition affecting public health. ${ }^{2}$ In the current coronavirus disease 2019 (COVID-19) climate, teachers have to complete long hours of online work, such as online lesson preparation, teaching, post-lesson question-and-answer sessions, and homework correction, thereby following the requirement by the Education Ministry of China to "stop classes without stopping teaching and learning".

Due to isolation at home because of COVID-19, eyeuse duration and intensity among teachers have increased significantly, causing a rise in the incidence of dry eye among these workers. Several studies have shown the increasing incidence and worsening symptoms of dry eye disease, but with no further exploration on treatment strategy. $^{3-5}$ Besides, the current treatment for dry eye, based on a lack of effective etiological and other comprehensive treatments, is primarily symptom relief. Accordingly, establishing comprehensive and standardized treatments for dry eye is imperative.

The present study aimed to evaluate the effect of comprehensive treatment on the ocular surface function and the visual quality of online teachers with a mild-tomoderate dry eye condition during the early stage of the outbreak and to provide evidence for the treatment of dry eye among this demographic.

\section{Subjects and Methods}

This was a retrospective cross-section study and approved by the ethics committee of Affiliated Hospital of Southwest Medical University (KY2020027). This study was conducted in accordance with the Declaration of Helsinki. All participants had signed informed consent for enrollment.

\section{Subjects}

Thirty secondary school online teachers (60 eyes) who had been diagnosed with a mild-to-moderate dry eye condition in our hospital from February to May 2020 were enrolled, including 15 males and 15 females, aged $38.12 \pm 6.74$ years. The diagnosis of a mild-to-moderate dry eye condition was based on the 2013 Expert Consensus on Clinical Management of Dry Eye. ${ }^{1}$ The inclusion criteria were as follows: (I) teachers who participated in online work during the outbreak; (II) patients with mild-to-moderate dry eye disease; and (III) patients with the best-corrected visual acuity of 5.0 in both eyes. The exclusion criteria were as follows: (I) patients with significant myopia; ${ }^{6}$ (II) patients with no history of wearing a bandage contact lens; ${ }^{7}$ (III) patients with history of eye surgery or trauma; (IV) patients with active inflammation of the eye; and (V) patients with refractive medium turbidity disease.

\section{Methods}

\section{Questionnaire}

The same physician in the outpatient clinic surveyed the study participants about the time duration, type of devices and habits in which they used their eyes on electronic products, and their sleep duration and conducted an ocular surface disease index (OSDI) questionnaire among the study participants before treatment and at the 2nd and 4th week after treatment, respectively. How electronic products were used referred to the type of electronic product, the duration of each continuous use period, and the distance between the screen and the eyes when using electronic products. "Eye-use duration" for electronic products refers to the average daily use duration, and "sleep duration" refers to the average daily hours of sleep.

\section{Visual Quality Inspection}

The modulation transfer function (MTF) cutoff, strehl ratio (SR), basal objective scatter index (OSI), tear film objective scatter index (TF-OSI) and total $\mathrm{OSI}^{8,9}$ were measured by the same physician in the outpatient clinic using the optical quality analysis system (OQAS) (Visiometrics Inc., Spain) with dual-channel technology; ${ }^{10}$ measurements were conducted before and at 2 and 4 weeks after treatment, respectively. The correlations between each indicator were analyzed by comparing the changes in each parameter before and after treatment at the 2 nd and the 4th week.

\section{Detection of the Indicators Related to Dry Eye}

The central tear meniscus height (TMH), non-invasive first tear film breakup time (NIBUTf), non-invasive average tear film breakup time (NIBUTav), Meibomian gland score, and eye redness analysis were performed for the participants by the same physician in the outpatient clinic using the Keratograph $5 \mathrm{M}$ ocular surface analyzer before treatment and at the 2nd and 4th week after treatment, respectively. The Meibomian gland score was calculated using the methods proposed by Arita both before and after treatment. ${ }^{11}$ Slitlamp microscope, ophthalmoscope and speciality check-up were used to exclude active eye inflammation, for ensuring the accuracy of eye redness analysis. 


\section{Comprehensive Treatment for Dry Eye}

The following approaches were provided to the study participants.

(I) Health education: To help the participants understand the natural course of dry eye and the chronic nature of the condition and involve them in its management to reduce recurrence and chronicity. (II) Etiological therapy: Several recommendations were provided that an eye-use session lasting 30-45 $\mathrm{min}$ be adopted, followed by a break of 5-10 min; A distance of at least $33 \mathrm{~cm}$ be maintained when using electronic products and that a screen shield or eye protection glasses be used alongside other precautions. (III) Psychological comfort and encouragement: To help patients to relieve anxiety and depression, enhance confidence, and improve compliance. Cellphone messages of intervention measures as reminders for patients and telephone follow-up were proceeded every week. The follow-up with guidance of psychological assessment and consult was proceeded by a psychologist. Psychological regulation was composed of emotional catharsis, meditation, muscle relaxing and active cognitive behavioral therapy. (IV) Lacrimal replacement therapy: Preservative-free $0.1 \%$ sodium glass acid eye drops 4 times per day. (V) Hot-compress therapy of the Meibomian glands: Under the guidance of a physician, a hot massage of the Meibomian glands should be performed at home to speed up local blood flow to the eyelids, soften the eyelid fat, improve blockage of the Meibomian duct, reduce Meibomian gland dysfunction, and reduce tear evaporation. (VI) Modification of eyelid exposure and blinking abnormalities: The height of the table and chair should match the height of the device, which should be placed at a position of $10^{\circ}$ to $20^{\circ}$ below the horizon, enabling the user to look down when using electronic products to reduce eye surface exposure and avoid tear evaporation. Patients should also consciously increase blinking frequency to promote the redistribution of tears and maintain the stability of the tear film. (VII) Environmental modification: Indoor lighting should be moderate, and the humidity of the air in the room should be increased; ventilation should be strengthened, and air-condition and smoking/exposure to a greasy/smoky environment should be avoided.

\section{Statistical Analysis}

All the data collected in this study were analyzed using SPSS Statistics (v.25.0) software. Normally distributed measurement data were expressed as mean \pm standard deviation $(\bar{X} \pm \mathrm{SD})$, while non-normally distributed measurement data were expressed as median (interquartile range), and the comparisons were examined by Student- $t$-test and MannWhitney test (non-parametric distribution). Analysis of variance of the one-way repeated measurement was conducted for making comparisons at different points before and after treatment. A post-hoc comparative analysis was adopted for the two-by-two comparisons between different time points. Pearson's correlation test was used to conduct a correlation analysis; $\mathrm{P}<0.05$ was considered statistically significant.

\section{Results}

\section{The Changes to Each Indicator Detected by the Keratograph $5 \mathrm{M}$ Ocular Surface Analyzer Before and After Treatment}

At the 2nd and 4th week after treatment, the TMH values of the central lower eyelid were $0.53 \pm 0.09$ and $0.51 \pm$ $0.08 \mathrm{~mm}$, respectively; these values were higher than the value of $0.10 \pm 0.03 \mathrm{~mm}$ before treatment with statistically significant difference $(\mathrm{P}<0.001)$. The NIBUTf values were $11.09 \pm 2.33$ and $11.68 \pm 1.9 \mathrm{~s}$ at the $2 \mathrm{nd}$ and the 4 th week after treatment, respectively, which were higher than the value of $2.62 \pm 0.53 \mathrm{~s}$ before treatment with a statistically significant difference $(\mathrm{P}<0.001)$. The NIBUTav values were $15.65 \pm 3.05$ and $15.66 \pm 3.12 \mathrm{~s}$ at the 2 nd and 4 th week after treatment, respectively, which were significantly higher than the value of $5.58 \pm 1.07 \mathrm{~s}$ before treatment $(\mathrm{P}<$ $0.001)$. The 2 nd and the 4 th week Meibomian gland scores after treatment were $2.15 \pm 1.13$ and $1.88 \pm 0.9$, respectively, which were significantly lower than the score of $3.80 \pm 1.12$ before treatment, with statistical significance $(\mathrm{P}<0.001)$. There were no statistical differences in conjunctival and ciliary congestion between the measurements before and after treatment $(\mathrm{P}=0.677$ and 0.372 , respectively). There was no difference in the TMH of the central lower eyelid, NIBUTf, NIBUTav, Meibomian gland score, and conjunctival and ciliary congestion between the 2nd and the 4th week after treatment $(P>0.05)$. The detailed results are shown in Table 1.

\section{The Changes in Each Indicator Detected by the Dual-Channel Optical Quality Analysis System Before and After Treatment}

After treatment, the 2 nd and the 4th week OSI values were $0.43 \pm 0.29$ and $0.41 \pm 0.34$, respectively, which were significantly lower than the value of $1.29 \pm 0.53$ before 
Table I The Comparison of Each Indicator Before and After Treatment Detected by the Keratograph 5M Ocular Surface Analyzer

\begin{tabular}{|l|c|c|c|c|c|}
\hline Indicators & Before Treatment & After Treatment 2nd W & After Treatment 4th W & F & P-value \\
\hline TMH & $0.10 \pm 0.03$ & $0.53 \pm 0.09^{*}$ & $0.51 \pm 0.08^{*}$ & 1553.013 & $<0.001$ \\
First tear film breakup time & $2.62 \pm 0.53$ & $11.09 \pm 2.33^{*}$ & $11.68 \pm 1.9^{*}$ & 2652.831 & $<0.00 \mathrm{I}$ \\
Average tear film breakup time & $5.58 \pm 1.07$ & $15.65 \pm 3.05^{*}$ & $15.66 \pm 3.12^{*}$ & 740.460 & $<0.00 \mathrm{I}$ \\
Meibomian gland score & $3.80 \pm 1.12$ & $2.15 \pm 1.13^{*}$ & $1.88 \pm 0.9 *$ & 74.613 & $<0.00 \mathrm{I}$ \\
Conjunctival congestion & $0.56 \pm 0.19$ & $0.53 \pm 0.2$ & $0.56 \pm 0.21$ & $0.39 \mathrm{I}$ & 0.677 \\
Ciliary congestion & $0.53 \pm 0.19$ & $0.50 \pm 0.21$ & $0.56 \pm 0.21$ & 0.997 & 0.372 \\
\hline
\end{tabular}

Note: *Indicated statistically significant difference when compared with those before the treatment $(p<0.05)$.

Abbreviations: $\mathrm{TMH}$, tear meniscus height; $\mathrm{W}$, week.

treatment $(\mathrm{P}<0.001)$. The TF-OSI at the 2 nd and the 4 th week after treatment were $0.55 \pm 0.42$ and $0.51 \pm 0.37$, respectively, which were significantly lower than the value of $1.48 \pm 0.56$ before treatment $(\mathrm{P}<0.001)$. The MTF cutoff values were $44.19 \pm 4.12$ and $44.20 \pm 3.97$ at the 2nd and the 4th week after treatment were significantly higher than the value of $31.89 \pm 2.58$ before treatment $(\mathrm{P}<$ $0.001)$, respectively. The SR values at the 2 nd and the 4 th weeks after treatment were $0.27 \pm 0.2$ and $0.28 \pm 0.197$, respectively, which were significantly higher than the value of $0.20 \pm 0.17$ before treatment $(\mathrm{P}<0.001)$. The differences in OSI, TF-OSI, MTF cutoff, and SR at the 2nd and the 4th week after treatment were with no statistical significance $(\mathrm{P}>0.05)$. Detailed information mentioned above are shown in Table 2.

\section{The Changes in Each Indicator Based on the Questionnaire}

As shown in Table 3, the OSDI values were $6.13 \pm 2.66$ and $5.87 \pm 2.71$ at the 2 nd and the 4 th week after treatment, which were significantly lower than the value of $23.1 \pm 4.55$ before treatment $(\mathrm{P}<0.001)$, respectively. At the 2 nd and the 4 th weeks after treatment, the eye-use duration values were $3.36 \pm 2.01$ and $3.49 \pm 1.87 \mathrm{~h}$, which were significantly lower than the value of $9.20 \pm 1.69$ before treatment $(\mathrm{P}<0.001)$. There was no difference in sleep duration before and after treatment $(P=0.241)$. The differences in the OSDI, eye-use duration, and sleep duration between the 2nd and the 4th week after treatment were with no statistical significance $(\mathrm{P}>0.05)$.

\section{The Correlation Between Tear Film Objective Scatter Index and Each Indicator Before and After Treatment}

Before treatment and at the 2nd and the 4th weeks after treatment, the TF-OSI was positively correlated with OSDI, Meibomian gland scores, and eye-use duration with statistically significant difference $(\mathrm{P}<0.05)$, while it was negatively correlated with NIBUTf, NIBUTav, the

Table 2 Comparison of OQAS-II Objective Visual Quality Indicators Before and After Treatment with Dual-Channel Technology

\begin{tabular}{|l|c|c|c|c|c|}
\hline Indicators & Before Treatment & After Treatment 2nd W & After Treatment 4th W & F & P-value \\
\hline OSI & $1.29 \pm 0.53$ & $0.43 \pm 0.29^{*}$ & $0.41 \pm 0.34^{*}$ & 638.980 & $<0.001$ \\
TF-OSI & $1.48 \pm 0.56$ & $0.55 \pm 0.42^{*}$ & $0.51 \pm 0.37^{*}$ & 729.017 & $<0.001$ \\
MTF cut off & $31.89 \pm 2.58$ & $44.19 \pm 4.12^{*}$ & $44.20 \pm 3.97^{*}$ & 1931.942 & $<0.001$ \\
SR & $0.20 \pm 0.17$ & $0.27 \pm 0.2^{*}$ & $0.28 \pm 0.19^{*}$ & 116.819 & $<0.001$ \\
\hline
\end{tabular}

Note: *Indicated statistically significant difference when compared with those before the treatment $(p<0.05)$.

Abbreviations: OSI, objective scatter index; TF-OSI, tear film objective scatter index; MTF, modulation transfer function; SR, strehl ratio; W, week.

Table 3 The Comparison of Each Indicator Before and After Treatment by Questionnaire

\begin{tabular}{|l|c|c|c|c|c|}
\hline Indicators & Before Treatment & After Treatment 2nd W & After Treatment 4th W & F & P-value \\
\hline OSDI & $23.1 \pm 4.55$ & $6.13 \pm 2.66^{*}$ & $5.87 \pm 2.7 I^{*}$ & 2328.766 & $<0.001$ \\
Eye-use duration & $9.20 \pm 1.69$ & $3.36 \pm 2.0 I^{*}$ & $3.49 \pm 1.87^{*}$ & 1334.532 & $<0.00 \mathrm{I}$ \\
Sleep duration & $8.05 \pm 2.35$ & $8.07 \pm 2.31$ & $8.21 \pm 1.70$ & 1.459 & $0.24 \mathrm{I}$ \\
\hline
\end{tabular}

Note: *Indicated statistically significant difference when compared with those before the treatment $(p<0.05)$.

Abbreviations: OSDI, ocular surface disease index; W, week. 
Table 4 The Correlation of TF-OSI and Each Indicator Before and After Treatment

\begin{tabular}{|c|c|c|c|c|c|c|}
\hline \multirow[t]{2}{*}{ Indicators } & \multicolumn{2}{|c|}{ Before Treatment } & \multicolumn{2}{|c|}{ After Treatment 2nd W } & \multicolumn{2}{|c|}{ After Treatment 4th W } \\
\hline & $\mathbf{r}$ & P-value & $\mathbf{r}$ & P-value & $\mathbf{r}$ & P-value \\
\hline Meibomian gland score & 0.453 & $<0.001$ & 0.921 & $<0.001$ & 0.902 & $<0.001$ \\
\hline Eye-use duration & 0.957 & $<0.001$ & 0.948 & $<0.001$ & 0.947 & $<0.001$ \\
\hline First tear film breakup time & -0.624 & $<0.001$ & -0.529 & $<0.001$ & -0.518 & $<0.001$ \\
\hline Average tear film breakup time & -0.489 & $<0.001$ & -0.353 & $<0.001$ & -0.405 & 0.001 \\
\hline $\mathrm{TMH}$ & -0.705 & $<0.001$ & -0.545 & $<0.001$ & -0.548 & $<0.001$ \\
\hline Sleep duration & -0.383 & $<0.001$ & -0.328 & 0.011 & -0.316 & 0.014 \\
\hline Conjunctival congestion & -0.086 & 0.513 & -0.065 & 0.621 & -0.288 & 0.026 \\
\hline Ciliary congestion & -0.037 & 0.779 & 0.064 & 0.625 & 0.041 & 0.753 \\
\hline OSI & 0.850 & $<0.001$ & 0.856 & $<0.001$ & 0.861 & $<0.001$ \\
\hline MTF cut off & -0.754 & $<0.001$ & -0.723 & $<0.001$ & -0.732 & $<0.001$ \\
\hline SR & -0.743 & $<0.001$ & -0.720 & $<0.001$ & -0.753 & $<0.001$ \\
\hline
\end{tabular}

Abbreviations: TMH, tear meniscus height; OSI, objective scatter index; MTF, modulation transfer function; SR, strehl ratio; W, week.

$\mathrm{TMH}$ of the central lower eyelid, SR, sleep duration, conjunctival congestion and the MTF cutoff with statistical significance $(\mathrm{P}<0.05)$. There was no correlation between TF-OSI and ciliary congestion $(\mathrm{P}>0.05)$. These detailed data are shown in Table 4.

\section{Discussion}

With the modern accelerated pace of life and the popularity of computers, tablets, cell phones, and other electronic products, the incidence of dry eye is increasing annually. During the COVID-19 pandemic, more people have been forced to work and study at home, which would induce high prevalence of dry eye disease, ${ }^{12}$ including teachers in China. In the present study, thirty participants underwent comprehensive treatment for their dry disease with significant improvement in symptoms and visual indicators compared with those before. At 2 and 4 weeks after treatment, the OSI and the TF-OSI were significantly lower than those before treatment. TF-OSI was positively correlated with the OSDI, the Meibomian gland scores, the eye-use duration, and the OSI, while it was negatively correlated with the NIBUTf, NIBUTav, the TMH of the central lower eyelid, the SR, and the MTF cutoff, which was consistent with previous studies. $^{13,14}$

Comprehensive treatment should be implemented for dry eye patients rather than symptom relief only. Health education was recommended as an important aspect of successful treatment for dry eye. ${ }^{15}$ Besides, the incidence of anxiety and depression in patients with dry eye is higher than that in healthy population. ${ }^{16}$ Moreover, dry eye has been proved and defined as a chronic and progressive disease, ${ }^{17,18}$ of which etiologies are complex and may not be completely cured. ${ }^{2}$ Moon et al suggested that the number of eye blinks among prolonged video terminal workers was only $1 / 3$ of that in the normal population, and it would lead to a lack of the timely application of tears to the ocular surface, resulting in a decrease in the stability of the local TF and causing dry eye. ${ }^{1}$ Additionally, horizontal forward fixation of the eyes when using electronic devices would significantly increase the eyes' exposure down to the ocular surface, compared with normal reading, which would (indirectly) lead to the rapid evaporation of tears from the ocular surface. ${ }^{19,20}$ Furtherly, for the special circumstances during the COVID-19 pandemic, online teachers were quarantined at home and limited in terms of outdoor exercise would have more risk on incidence of dry eye. ${ }^{21}$ Thus, comprehensive treatment should be provided to maximize quality of life and curation of dry eye disease for patients. ${ }^{22}$ In the present study, a comprehensive treatment strategy composed of health education, etiology prevention, psychological comfort, lacrimal replacement, hot compress, modification of eyelid exposure, and environmental modification were provided, and significant improvements were obtained.

It is considerable for the teachers and the other professions to adopt our treatment strategy for their eye protection and dry eye treatment in daily lives. According to a requirement by the Ministry of Education in China, teachers had to complete work in form of online by increased electronic device usage. The incidence of dry eye increased significantly when electronic devices were used for more than $8 \mathrm{~h} /$ day. ${ }^{13}$ In addition, Tong et al and Şimşek et al showed that dry eye among video terminal 
operators correlated with dysfunction of the Meibomian glands and that reduced blinking frequency among these workers was an underlying pathophysiological mechanism for the high incidence of Meibomian gland disease. ${ }^{23,24}$ Recently, it was also suggested that three major factors, including a lack of outdoor exercise, prolonged sedentary activity, and the use of electronic devices, reduced tear production and affected the progression of dry eye, ${ }^{25}$ which was also previously reported..$^{21,26}$ Thus, not only teachers but also students and all professions with long time usage of electronic devices should take dry eye disease seriously and pay attention to the prevention of disease.

In the present study, following a comprehensive treatment for dry eye, the questionnaire results showed that the time duration of using electronic devices decreased from $9.20 \pm 1.69 \mathrm{~h}$ before treatment to $3.36 \pm 2.01 \mathrm{~h}$ after treatment. Additionally, dry eye-related indicators including the OSDI, Meibomian gland score, NIBUTf, NIBUTav, and the TMH of the central lower eyelid improved significantly at the 2 nd and the 4 th week after treatment, compared with the ocular surface function indicators before treatment. Conjunctival congestion and ciliary congestion were found to be important indicators of local inflammation of the ocular surface. Our findings showed no significant differences in bulbar conjunctival and ciliary congestion before and after treatment, which may be correlated with the small number of subjects and the limited data collection conducted for the present study. The visual quality indicators of OSI, MTF cutoff, SR, and the TF-OSI in patients with dry eye also improved significantly after treatment, compared with before treatment. It did confirm the effectiveness of our treatment strategy.

The TF-OSI may reflect the stability of the tear film in patients with dry eye, and the value of this factor correlates with the severity of dry eye. In the correlation analysis between TF-OSI and visual quality indicators, TF-OSI positively correlated with OSDI, Meibomian gland scores, eyeuse duration, and OSI, while it was negatively correlated with NIBUTf, NIBUTav, TMH of the central lower eyelid, SR, and MTF cutoff. These differences were statistically significant. These results were consistent with previous studies. $^{9,14}$

There were also several limitations of the present study. First, only 30 participants restricted to teachers were enrolled into this analysis for the short duration of online teaching in China. More patients in other professions should be enrolled into analysis in the future. Second, due to the restriction of treatment for out-clinic patients, compliance of patient treatment could not be evaluated accurately, while only cell phone message and telephone follow-up were proceeded. It should be improved by some quantifiable indicators to judge the compliance of out-clinic patients in further study. Third, the follow-up time was only 4 weeks, and more visual indicators and symptoms should be observed in the future. At last, for its nature of cross-section analysis, the decreased time duration of using electronic device would result in relieved symptoms and better indicator index. Cohort studies or randomized controlled trials should be done to further evaluate the effects of comprehensive treatment, if possible.

In conclusion, comprehensive treatment could effectively improve the stability of the tear film and the condition of the ocular surface for dry eye patients. It could also alleviate the symptoms and improve visual quality of dry eye during the early stage of the outbreak among online teachers who experienced a mild-to-moderate dry eye condition.

\section{Disclosure}

The authors report no conflicts of interest in this work.

\section{References}

1. Moon JH, Kim KW, Moon NJ. Smartphone use is a risk factor for pediatric dry eye disease according to region and age: a case control study. BMC Ophthalmol. 2016;16(1):188. doi:10.1186/s12886-0160364-4

2. Liu ZG, Wang H. [Focusing on the management of chronic dry eye disease]. Zhonghua Yan Ke Za Zhi. 2018;54(2):81-83. Chinese. doi:10.3760/cma.j.issn.0412-4081.2018.02.001

3. García-Ayuso D, Di Pierdomenico J, Moya-Rodríguez E, ValienteSoriano FJ, Galindo-Romero C, Sobrado-Calvo P. Assessment of dry eye symptoms among university students during the COVID-19 pandemic. Clin Exp Optom. 2021;1-7. doi:10.1080/08164622. 2021.1945411

4. Saldanha IJ, Petris R, Makara M, Channa P, Akpek EK. Impact of the COVID-19 pandemic on eye strain and dry eye symptoms. Ocul Surf. 2021;22:38-46. doi:10.1016/j.jtos.2021.06.004

5. Pandey SK, Sharma V. Mask-associated dry eye disease and dry eye due to prolonged screen time: are we heading towards a new dry eye epidemic during the COVID-19 era? Indian J Ophthalmol. 2021;69 (2):448-449. doi:10.4103/ijo.IJO_3250_20

6. Yotsukura E, Torii H, Inokuchi M, et al. Current prevalence of myopia and association of myopia with environmental factors among schoolchildren in Japan. JAMA Ophthalmol. 2019;137(11):1233-1239. doi:10.1001/jamaophthalmol.2019.3103

7. Kojima T. Contact lens-associated dry eye disease: recent advances worldwide and in Japan. Invest Ophthalmol Vis Sci. 2018;59(14): Des102-Des108. doi:10.1167/iovs.17-23685

8. Ye F, Jiang F, Lu Y, et al. Objective optical assessment of tear-film quality dynamics in patients with meibomian gland dysfunction and aqueous-deficient dry eye optical quality changes in different dry eye subtypes. Indian J Ophthalmol. 2019;67(5):599-603. doi:10.4103/ijo. IJO_1278_18 
9. Su YD, Liang QF, Wang NL, Antoine L. [A study on the diagnostic value of tear film objective scatter index in dry eye]. Zhonghua Yan Ke Za Zhi. 2017;53(9):668-674. Chinese. doi:10.3760/cma.j. issn.0412-4081.2017.09.007

10. Herbaut A, Liang H, Rabut G, et al. Impact of dry eye disease on vision quality: an optical quality analysis system study. Transl Vis $\mathrm{Sci}$ Technol. 2018;7(4):5. doi:10.1167/tvst.7.4.5

11. Pinna A, Blasetti F, Zinellu A, Carru C, Solinas G. Meibomian gland dysfunction and hypercholesterolemia. Ophthalmology. 2013;120 (12):2385-2389. doi:10.1016/j.ophtha.2013.05.002

12. Mohan A, Sen P, Shah C, Jain E, Jain S. Prevalence and risk factor assessment of digital eye strain among children using online e-learning during the COVID-19 pandemic: digital eye strain among kids (DESK study-1). Indian $J$ Ophthalmol. 2021;69 (1):140-144. doi:10.4103/ijo.IJO_2535_20

13. Inomata $\mathrm{T}$, Iwagami $\mathrm{M}$, Nakamura $\mathrm{M}$, et al. Characteristics and risk factors associated with diagnosed and undiagnosed symptomatic dry eye using a smartphone application. JAMA Ophthalmol. 2020;138 (1):58-68. doi:10.1001/jamaophthalmol.2019.4815

14. Gao Y, Liu R, Liu Y, et al. Optical quality in patients with dry eye before and after treatment. Clin Exp Optom. 2021;104(1):101-106. doi:10.1111/cxo.13111

15. Akpek EK, Amescua G, Farid M, et al. Dry eye syndrome preferred practice pattern ${ }^{\circledR}$. Ophthalmology. 2019;126(1):P286-P334. doi:10. 1016/j.ophtha.2018.10.023

16. Bitar MS, Olson DJ, Li M, Davis RM. The correlation between dry eyes, anxiety and depression: the sicca, anxiety and depression study. Cornea. 2019;38(6):684-689. doi:10.1097/ico.0000000000001932

17. Tsubota K, Yokoi N, Shimazaki J, et al. New perspectives on dry eye definition and diagnosis: a consensus report by the Asia dry eye society. Ocul Surf. 2017;15(1):65-76. doi:10.1016/j.jtos.2016.09.003
18. Nelson JD, Craig JP, Akpek EK, et al. TFOS DEWS II introduction. Ocul Surf. 2017;15(3):269-275. doi:10.1016/j.jtos.2017.05.005

19. Rico-Del-Viejo L, Benítez-Del-Castillo JM, Gómez-Sanz FJ, García-Montero M, Llorens-Quintana C, Madrid-Costa D. The influence of meibomian gland loss on ocular surface clinical parameters. Cont Lens Anterior Eye. 2019;42(5):562-568. doi:10.1016/j. clae.2019.04.004

20. Chhadva P, Goldhardt R, Galor A. Meibomian gland disease: the role of gland dysfunction in dry eye disease. Ophthalmology. 2017;124 (11):S20-S26. doi:10.1016/j.ophtha.2017.05.031

21. Kawashima M, Uchino M, Yokoi N, et al. The association between dry eye disease and physical activity as well as sedentary behavior: results from the Osaka Study. J Ophthalmol. 2014;2014:943786. doi:10.1155/2014/943786

22. Kawashima M. Systemic health and dry eye. Invest Ophthalmol Vis Sci. 2018;59(14):Des138-Des142. doi:10.1167/iovs.17-23765

23. Tong L, Teo CHY, Lee RKJ. Spatial distribution of noninvasive break up times and clinical relevance in healthy participants and mild dry eye. Transl Vis Sci Technol. 2019;8(5):30. doi:10.1167/ tvst.8.5.30

24. Şimşek C, Doğru M, Kojima T, Tsubota K. Current management and treatment of dry eye disease. Turk $J$ Ophthalmol. 2018;48 (6):309-313. doi:10.4274/tjo.69320

25. Hanyuda A, Sawada N, Uchino M, et al. Physical inactivity, prolonged sedentary behaviors, and use of visual display terminals as potential risk factors for dry eye disease: JPHC-NEXT study. Ocul Surf. 2020;18(1):56-63. doi:10.1016/j.jtos.2019.09. 007

26. Schmid D, Leitzmann MF. Television viewing and time spent sedentary in relation to cancer risk: a meta-analysis. $J$ Natl Cancer Inst. 2014;106(7). doi:10.1093/jnci/dju098
International Journal of General Medicine

\section{Publish your work in this journal}

The International Journal of General Medicine is an international, peer-reviewed open-access journal that focuses on general and internal medicine, pathogenesis, epidemiology, diagnosis, monitoring and treatment protocols. The journal is characterized by the rapid reporting of reviews, original research and clinical studies

\section{Dovepress}

across all disease areas. The manuscript management system is completely online and includes a very quick and fair peer-review system, which is all easy to use. Visit http://www.dovepress.com/ testimonials.php to read real quotes from published authors. 